\title{
Analyzing the dynamics of free recall: An integrative review of the empirical literature
}

\author{
JOHN T. WIXTED and DOUG ROHRER \\ University of California, San Diego, La Jolla, California
}

\begin{abstract}
Relatively few experiments have measured the time course of free recall from episodic or semantic memory. Of those that have, most report that cumulative recall is a negatively accelerated exponential (or hyperbolic) function that is characterized by two properties: asymptotic recall and rate of approach to asymptote. The most common measure of free recall performance (viz., the number of items recalled) provides a reasonably good estimate of asymptotic recall if a relatively long recall period is used (which is rare), but the effect of experimental manipulations on the rate of approach to asymptote cannot be determined without timing when recall responses occur. The research reviewed herein suggests that the rate of approach to asymptote may offer an estimate of the breadth of search through long-term memory. The search in question, unlike most of those investigated in the memory literature, is unique in that it requires minutes rather than milliseconds to complete.
\end{abstract}

When subjects attempt to generate items from a semantic category or to recall a recently presented list of words, they do not complete the task in an instant. Instead, their performance is almost invariably characterized by intermittent successes occurring over an extended period of time. In spite of this interesting fact, memory researchers have exhibited an overwhelming preference for recording which (or how many) items are recalled during some brief period of time, regardless of the speed with which those items have been retrieved. Does the time course of free recall offer any useful information about the nature of retrieval? Because this subject is an old one that commands little attention today, one might assume that the answer is no. However, a review of the extant literature, limited as it is, suggests otherwise.

Exactly what can be learned that is not already known by studying the dynamics of free recall? First, the studies that have tracked the time course of recall usually reveal that subjects continue to make progress far beyond the 1 - or 2-min recall period typically provided. Indeed, Roediger and Thorpe (1978)-to take one example that will be considered in detail later-reported that recall on an episodic memory task continued to increase more than 20 min into the recall period. Moreover, a clear difference in the level of recall between two conditions (easily named pictures vs. words) became evident only after several minutes had elapsed. Had these authors used the standard brief recall period, they might have mistakenly concluded that the two conditions produced equal levels of performance. How often are incorrect conclusions arrived at because the recall period is too brief? The answer is

Correspondence concerning this article should be sent to John Wixted, Department of Psychology-0109, University of California, San Diego, La Jolla, CA 92093. unknown because most researchers simply ignore the potential problem.

Second, most of the literature reviewed below suggests that the time course of free recall, like reaction time in the Sternberg memory scanning procedure, reflects the dimensions of search through memory. Unlike most memory search processes that take place on the order of milliseconds, however, the search of interest here requires minutes to complete. What variables affect the time course of this search process? Even the most experienced memory researcher may have difficulty answering this question because the sporadic research concerned with the timing of free recall has never been systematically analyzed.

\section{BACKGROUND}

Bousfield and Sedgewick (1944) initiated modern inquiry into this subject by measuring the dynamics of retrieval from semantic memory. In their experiment, subjects were asked to write down the names of as many items as possible from specific categories (e.g., birds, U.S. cities, etc.) for $18 \mathrm{~min}$. In addition, every $2 \mathrm{~min}$, the subjects were instructed to draw a line after the most recently recalled item. When the cumulative number of items recalled was plotted as a function of time, the resulting data revealed that recall did not progress in linear fashion but instead slowed continuously, with the maximum rate of recall occurring at the beginning of the recall period.

Bousfield and Sedgewick (1944) observed that their cumulative recall functions were reasonably well described by an exponential of the form

$$
F(t)=N\left(1-e^{-\lambda t}\right),
$$

where $F(t)$ represents the cumulative number of items recalled by time $t, N$ represents the number of items recalled given unlimited time (i.e., asymptotic recall), and $\lambda$ rep- 
resents the rate of approach to that asymptote. Note that $1 / \lambda$ is equal to the average latency to recall associated with the $N$ items that are ultimately recalled. Thus, a rapid rate of approach to asymptote (i.e., a large $\lambda$ ) implies a short average latency to retrieval, and vice versa. The exponential's main rival for describing cumulative recall is the hyperbola, which can be written as

$$
F(t)=N^{2} \lambda t /(1+N \lambda t),
$$

where $N$ and $\lambda$ have basically the same interpretation as the corresponding parameters in the exponential (Bousfield, Sedgewick, \& Cohen, 1954).

Figure 1 presents cumulative free recall data taken from one of the conditions (pleasant activities) reported by Bousfield and Sedgewick (1944), along with the bestfitting exponential. This figure, which shows the cumulative number of items recalled up to each point in the recall period, clearly illustrates the two properties that characterize the time course of free recall: asymptotic recall (indicated by the dashed line) and rate of approach to asymptote, which in this case is rather gradual. Experimental manipulations that affect one property of recall may or may not affect the other for reasons that turn out to be theoretically interesting.

Bousfield and Sedgewick (1944) and McGill (1963) noted that the form of Equation 1 is consistent with a random search model according to which individual items are randomly sampled from a search set, evaluated, and then replaced. The gradual slowing of recall, according to this account, arises because of the resampling of al-

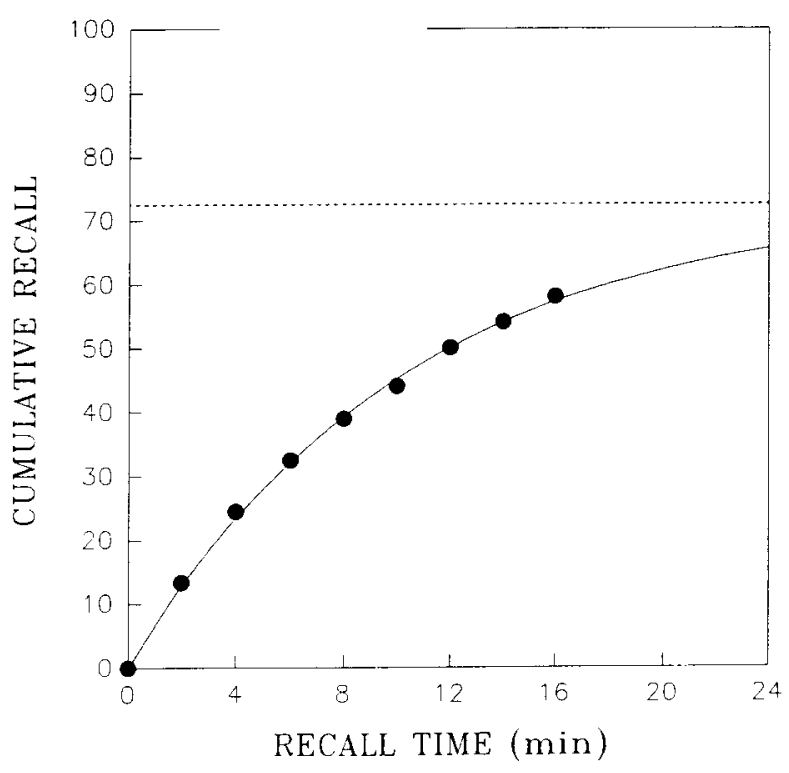

Figure 1. A cumulative free recall latency distribution taken from Bousfield and Sedgewick (1944). The data represent the cumulative number of pleasant activities generated by 18 subjects as a function of time. The solid curve represents the best-fitting exponential (Equation 1), and the dashed line indicates the asymptotic level of recall. ready retrieved items. That is, early in the recall period, almost every draw from the search set yields a new item (which is overtly recalled). Later, after most of the items have been recalled, almost every draw retrieves an already sampled item (which is not overtly recalled). Various modifications of this model will yield hyperbolic, instead of exponential, recall. Bousfield et al. (1954), for example, noted that if recalling an item makes that item more likely to be sampled again (and correspondingly decreases the likelihood that not-yet-retrieved items are sampled), then a hyperbolic growth curve might be expected (cf. Indow \& Togano, 1970).

Most of the initial research into the dynamics of recall followed the lead of Bousfield and Sedgewick by studying retrieval from semantic memory, with studies involving episodic memory generally appearing somewhat later. In roughly chronological order, the major issues addressed in this literature are (1) the relationship between the two parameters of the exponential (or hyperbolic) cumulative recall function, (2) the temporal dynamics of clustering in recall, (3) the role of cumulative recall in elucidating part-list cuing effects and hypermnesia, and (4) mathematical elaborations of the basic sampling-with-replacement search model.

\section{THE APPARENT RELATIONSHIP BETWEEN $\boldsymbol{N}$ and $\lambda$}

\section{Semantic Memory}

One of the most interesting facts about retrieval from semantic memory concerns the relationship between $N$ (asymptotic recall) and $\lambda$ (rate of approach to asymptote). Johnson, Johnson, and Mark (1951), who first observed that the two parameters were related, asked subjects to generate as many items as possible from specific semantic categories (cities first, then animals). When Equation 1 was fit to the data from individual subjects, these authors found that $N$ and $\lambda$ were strongly negatively correlated. For cities, the correlation was -.78; for animals, the corresponding value was -.48 . Thus, subjects who were able to name a larger number of cities approached their higher asymptotes more slowly than did subjects who could name fewer cities.

Kaplan, Carvellas, and Metlay (1969) showed that the inverse correlation between $N$ and $\lambda$ did not arise from individual differences between subjects because it also $o c$ curs within individuals exposed to different conditions. In their experiment, each subject was asked to generate as many 4-letter words as possible from a pool of letters ranging from 5 to 10 across conditions. The number of words produced as a function of time within each condition was well described by Equation 1 . Obviously, $N$ increased as the pool of letters to draw from increased. Less obviously, $\lambda$ decreased under the same conditions. Thus, when subjects could produce only a few 4-letter words (e.g., from 5-letter sets), performance approached asymptote rapidly. When those same subjects could produce many 4-letter words (e.g., from 10-letter sets), performance approached asymptotic recall more slowly. 
Herrmann and Chaffin (1976) investigated whether or not the same result would hold true across taxonomic categories. That is, do subjects generate items at a proportionately slower rate from large semantic categories than from smaller ones? To address this question, subjects were asked to generate items from 12 different semantic categories. When Equation 1 was fit to the group data for each category, the obtained correlation between $N$ and $\lambda$ was -.51 in one experiment and -.75 in another. Herrmann and Murray (1979) replicated this result using large categories (e.g., bodies of water) versus smaller nested categories (e.g., lakes). Figure 2 shows the average data from this experiment along with the best-fitting exponential. For both categories, performance was clearly still increasing by the end of the recall period (which is often true of episodic memory experiments as well). The estimated values of $N$ and $\lambda$ based on a least squares fit of Equation 1 to the large category data were 22.4 and 0.52 , respectively, whereas the corresponding values for the small categories were 15.0 and 0.84 .

Why should $N$ and $\lambda$ be negatively correlated? One relatively uninteresting, but nevertheless plausible, explanation is that subjects who could generate more items began retrieving those items at a faster rate than they could be written down or spoken aloud. If so, then rapidly recalled words would begin to "queue up" in short-term store, thereby delaying their overt retrieval. The effect would be to artifactually reduce the estimated value of $\lambda$. Johnson et al. (1951), who first discovered the relationship in question, considered this to be an unlikely possibility because the fastest recall rates rarely exceeded 20 words per minute (well below the maximum rate) and recall totals were measured at 3-min intervals. By the time 1

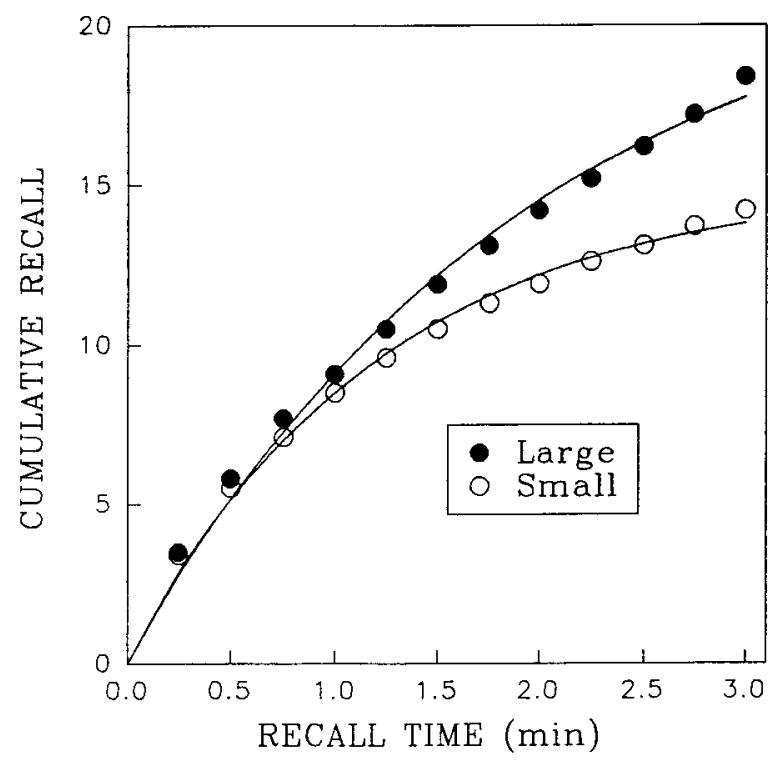

Figure 2. Cumulative free recall functions for large and small semantic categories from Herrmann and Murray (1979). The solid curves represent the best-fitting exponential functions (Equation 1). or $2 \mathrm{~min}$ had elapsed, recall rates were usually quite low. Thus, it seems unlikely that any words were still queued after the first interval. Instead, they argued that the limiting factor was apparently cognitive in nature.

The inverse correlation between $N$ and $\lambda$ implies a direct correlation between $N$ and recall latency (i.e., as more items are retrieved, average recall latency increases). Considered in that light, almost any retrieval scheme would predict the obtained result. Consider, for example, a very simple model that assumes that items in semantic memory are scanned in linear fashion (as in the Sternberg shortterm memory task). If the scan rate were one item per second and the category contained only one item (i.e., $N=1$ ), then average recall latency would be $1 \mathrm{sec}$. If the category contained two items (i.e., $N=2$ ), then average recall latency would increase to $1.5 \mathrm{sec}$ because the first item would be recalled after $1 \mathrm{sec}$ and the second after $2 \mathrm{sec}$. The larger the search set, the longer the average latency to recall.

The linear scan model, while consistent with the observed relationship between $N$ and recall latency, seems inconsistent with the other major empirical finding from this literature-namely, the exponential form of retrieval. A linear scan model anticipates a linear approach to asymptote rather than the negatively accelerated approach typically observed. As indicated earlier, an alternative model long known to be consistent with exponential retrieval, and which also predicts the inverse correlation between $N$ and $\lambda$, assumes random sampling with replacement from a finite search set. According to this account, the boundaries of the search are established by a retrieval cue (e.g., "generate cities"), and items are overtly recalled the first time they are sampled.

McGill (1963) showed that this sampling scheme predicts exponential retrieval and, more to the point, that it necessarily predicts the following inverse relationship between search set size and $\lambda$ :

$$
\lambda=r / S,
$$

where $r$ is the rate at which items are sampled from a search set of size $S$. Because mean recall latency $(\tau)$ is equal to $1 / \lambda$, the random search model also implies that $\tau=S / r$. In other words, assuming a constant rate of sampling, mean latency grows linearly with the size of the search set (a point that was also true of the linear scan model discussed above). A similar relationship between $\lambda$ and $S$ exists for the simplest hyperbolic sampling-withreplacement model discussed earlier-namely, $\lambda=r / S^{2}$ (cf. Bousfield et al., 1954). Table 1 presents a reference list of definitions for the mathematical symbols used throughout this article. Note that $\lambda, \tau$, and $N$ are empirical estimates, whereas $r$ and $S$ represent theoretical quantities.

In all of the studies described above, asymptotic recall (i.e., $N$ ) was inversely related to rate of approach to asymptote $(\lambda)$, but the random search model suggests that, in theory, it is actually search set size (i.e., $S$ ) that is inversely related to $\lambda$ (cf. Equation 3). According to this 
Table 1

Definitions of Mathematical Symbols

\begin{tabular}{cl}
\hline Symbol & \multicolumn{1}{c}{ Definition } \\
\hline$\lambda$ & Rate of Approach to Asymptote \\
$\tau$ & Mean Recall Latency $(1 / \lambda)$ \\
$\mathrm{N}$ & Asymptotic Number of Items Recalled \\
$\mathrm{r}$ & Rate of Sampling from Search Set* \\
$\mathrm{S}$ & Number of Representations in Search Set* \\
\hline
\end{tabular}

*Theoretical measures.

model, the reason why $N$ and $\lambda$ are inversely related is because, usually, $N$ and $S$ are equivalent (or, at least, directly related). Consider, for example, two semantic categories, female names and signs of the zodiac. The former category is presumably associated with a vastly larger search set than the latter (i.e., there are many more female names to sample and replace than there are signs of the zodiac). As a result, $\lambda$ for female names should be small. In addition, many more female names will be overtly generated than signs of the zodiac (i.e., $N$ will be larger for female names as well). Thus, a larger $N$ in this case implies a larger $S$.

If conditions are arranged such that $N$ no longer provides a reasonable estimate of $S$, then, according to the random search model, the usual inverse correlation between $N$ and $\lambda$ should disappear. By contrast, any theory that assumes that $N$ and $\lambda$ are intrinsically inversely related (e.g., because of limitations on motor output) would predict the usual inverse relationship regardless of whether or not $N$ is equal to $S$. Metlay, Handley, and Kaplan (1971) performed an experiment relevant to this issue. Subjects were asked to generate items from particular categories (e.g., Presidents) that were further subcategorized into sets of various sizes. In one condition, for example, subjects were instructed to generate the names of Presidents that contained the letter $y(N=5)$. In other conditions, the names had to contain the letter $s(N=11)$ or the letter $e(N=15)$. Presumably, subjects in each condition were forced to silently generate the names of 32 Presidents while overtly reporting only those that satisfied the specific letter requirement. Thus, this procedure should hold the overall size of the mental search set constant ( $S=32$ ) while varying the number of target items in that search set. Nevertheless, the time course of retrieval should still be exponential and (because of Equation 3) $\lambda$ should remain constant in spite of differences in $N$. On the other hand, if $N$ and $\lambda$ were inversely correlated in previous experiments because of the extra motor time required to produce items when $N$ is large, then this experiment, like its predecessors, should produce the usual inverse correlation. In accordance with the search model, Metlay et al. (1971) found that $\lambda$ remained constant in spite of large changes in $N$.

This result is instructive when considered in relation to those reported by Herrmann and Murray (1979). When subjects were asked to generate items from nested semantic categories, the usual inverse relation between $N$ and $\lambda$ was observed. This implies that when asked to gener- ate lakes, for example, subjects do not create a search set comprised of "bodies of water" and select lakes from that set but instead create a more focused search set (as one would expect). That is, presumably, lakes are stored as lakes. On the other hand, when asked to generate nested items that contain a particular letter (such as Presidents' names containing $y$ ), the usual inverse relationship is not observed, which implies only one search set regardless of the letter chosen. Presumably, Presidents' names are not stored according to the individual letters that comprise them.

The results reported by Metlay et al. (1971) suggest that, as required by Equation $3, \lambda$ is inversely related to $S$, not $N$. For the kind of experiment they performed, this result might seem to be trivially true. That is, the only reason $N$ and $S$ were not equal is because the instructions induced a conscious editing strategy that simply excluded certain covertly retrieved items from being overtly recalled. However, the random search model does not distinguish between dissociations of $N$ and $S$ accomplished through conscious editing (in which case, the result may seem trivial) or preconscious editing. In studies of semantic memory, it may not be possible to separate $N$ from $S$ without inducing conscious editing strategies, but the same does not appear to be true of studies involving episodic memory.

\section{Episodic Memory}

The relationship between asymptotic recall $(N)$ and rate of approach to asymptote $(\lambda)$ in studies of episodic memory is actually quite variable because, theoretically, the relationship between $N$ and the size of the search set $(S)$ is variable. For example, Wixted and Rohrer (1993) exposed subjects to lists of three items, followed by a distractor task, followed by a 20 -sec recall period during which verbal response latencies were timed to the nearest second. Blocks of three trials involved words from the same semantic category. The results of many previous studies suggest that asymptotic performance should decline with the buildup of proactive interference (i.e., across the three trials within a block), and that was indeed the obtained result. The values of $N$ on Trials 1,2 , and 3 were $2.62,2.30$, and 1.97 , respectively. Because $N$ decreases with the buildup of proactive interference (PI), and because $N$ and $\lambda$ are usually inversely correlated, one might expect $\lambda$ to increase across trials. In contrast to this prediction, the results showed a dramatic decrease in $\lambda$ with the buildup of PI $(0.35,0.20$, and 0.15 , for Trials 1,2 , and 3, respectively). In other words, asymptotic recall $(N)$ and rate of approach to asymptote $(\lambda)$ were positively correlated, which is exactly the opposite of the standard finding.

This result is actually predicted by Equation 3, which relates $\lambda$ to the size of the search set rather to value of the asymptote. Over the course of three trials involving words from the same semantic category, it seems reasonable to assume that the search set increases (Baddeley, 1990; Crowder, 1976; Watkins \& Watkins, 1975). For 
example, consider a block of three trials involving words from the category "sports." After the first trial, the search set established by the retrieval cue sports presumably includes the three words on the list. After the second trial, the same retrieval cue would presumably produce a search set that includes words from both the first and the second list. An even larger search set would be established after the third list of sports. Thus, if the buildup of PI is associated with a growing search set, as most theories of PI suggest, then Equation 3 requires that $\lambda$ decrease across trials (which it does). The reason why $N$ and $\lambda$ are not inversely correlated in this case is that $N$ does not provide an index of search set size (which was also true of the study by Metlay et al., 1971).

In a sense, the PI experiment described above can be construed as a list length experiment (Watkins \& Watkins, 1975). That is, although the nominal list length was always three items, the effective list length increased with the buildup of PI. This cue-overload interpretation implies that $\lambda$ should decrease as a function of increasing list length in episodic memory experiments. In addition, because longer lists will also be associated with higher asymptotic recall, the usual inverse association between $N$ and $\lambda$ should hold. Roediger and Tulving (1979) presented subjects with lists of 30 or 60 words, followed by a 10-min recall period. Although a mathematical analysis of the obtained cumulative recall curves was not performed, their data nevertheless revealed that performance in the 60 -word condition approached the higher asymptote at a slower rate than did performance in the 30-word condition. Fitting Equation 1 to these data (estimated from Roediger \& Tulving's Figure 4) revealed that $N$ increased from 18.2 to 31.1 and $\lambda$ decreased from 1.06 to 0.55 as list length increased from 30 to 60 words. Thus, the expected inverse relation between $N$ and $\lambda$ was obtained because, theoretically, $N$ and $S$ were directly related in this experiment.

Some additional experiments recently performed in our laboratory (Rohrer \& Wixted, in press), including a replication of the above result, are also most easily interpreted in terms of a model that assumes that the rate of approach to asymptote is inversely related to the size of the search set (and is not intrinsically related to the value of the asymptote). Subjects in one experiment were exposed to lists of three, six, or nine items (with each word presented for $2 \mathrm{sec}$ ) followed by a 20 -sec distractor task, followed by a free recall test. Thus, this experiment is basically a three-level replication of Roediger and Tulving's (1979) experiment, although the lists were much shorter. The obtained results were as expected: whereas the values of $N$ were $2.55,3.96$, and 4.68 for lists of three, six, and nine items, respectively, the corresponding values of $\lambda$ were $0.44,0.21$, and 0.16 . Note that these values of $\lambda$ are quite similar to those obtained from the PI experiment discussed above (namely, .35, .20, and .15 for Trials 1, 2, and 3, respectively) in which the effective list length was assumed to increase from three to six to nine items across trials, even though the nominal list length was always three items.
Subjects in a second experiment were exposed to lists of six items for varying lengths of study time. The words were presented at a rate of one every $4 \mathrm{sec}$ (slow), one every $2 \mathrm{sec}$ (medium), or one every $1 \mathrm{sec}$ (fast). Obviously, asymptotic recall decreased as the rate of presentation increased. For the slow, medium, and fast conditions, the obtained values of $N$ were 4.56, 3.60, and 3.00 words, respectively, while the corresponding values of $\lambda$ were $0.20,0.20$, and 0.19 . Stated differently, rate of approach to asymptote remained constant as the percentage of items recalled decreased from $76 \%$ to $50 \%$. In terms of the random search model, this result implies that duration of study does not affect the size of the search set (cf. Equation 3).

Why should the search set remain constant and $N$ change as the duration of study increases? Most theories of memory agree that the presentation of a word will create some representation (i.e., a trace to be sampled) that may or may not be sufficiently complete to reconstruct the original item. Using the terminology of Raaijmakers and Shiffrin (1980), sampling a representation may or may not lead to the recovery of the list item that created it. Such an idea seems reasonable because subjects can often provide details about items they cannot remember (e.g., what the first letter is, what the word rhymes with, etc.). Thus, in the study just described, the presentation of six words might create six representations to be searched (i.e., $S=6$ ), perhaps only three of which are sufficiently intact to recover the corresponding list item when sampled (i.e., $N=3$ ). A longer duration of study presumably creates a more intact memory trace, but not necessarily a greater number of memory traces. By contrast, presenting more items on a list will increase the total number of representations as well as the number of recoverable items. Thus, in that case, $N$ should increase because the absolute number of intact traces will increase and $\lambda$ should decrease because the total search set (composed of both intact and degraded traces) increases as well.

Figures 3 and 4 present graphical illustrations of this model for list length and rate of presentation, respectively. The figures show hypothetical search sets for the three conditions of each experiment. An $X$ represents an intact trace (i.e., one that will recover a list item when sampled), whereas an $O$ represents a degraded trace (i.e., one that will not recover a list item when sampled). Also shown are the numerical values of $S$ (search set size), $N$ (number of recoverable items in the search set, which equals asymptotic recall), and $p$ (the hypothetical probability that a list item is encoded in recoverable form). Note the value of $p$ is simply the asymptotic probability of recall (i.e., $N / S$ ), which is known to decrease with list length and increase with duration of study. Theoretically, the value of $\lambda$ should be inversely related to the values in the $S$ column, whereas asymptotic recall should be equal to the values in the $N$ column. This, of course, reflects the obtained pattern of results.

A similar conceptualization applies to the PI experiment discussed earlier. Indeed, the PI model is identical to the list length illustration in Figure 3 (see Watkins \& Wat- 


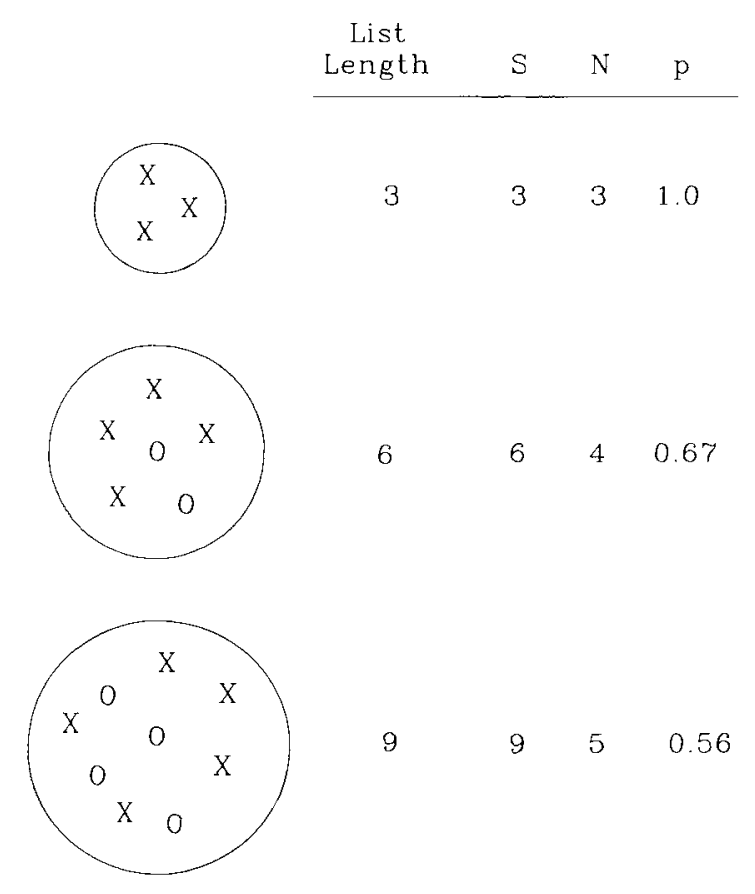

Figure 3. Hypothetical search set established by lists of three, six, and nine items (top, middle, and bottom, respectively). $X$ represents an intact representation, whereas $O$ represents a degraded representation. $S=$ total items in search set, $\boldsymbol{N}=$ number of intact items in search set, $p=$ probability that a list item is encoded in intact form.

kins, 1975), except that asymptotic recall decreases as the search set increases. Note that the models in Figures 3 and 4 (and the corresponding model for PI) predict a negative correlation, a zero correlation, and a positive correlation between $\lambda$ and $N$, respectively, although $\lambda$ is inversely related to the theoretical value of $S$ in all cases.

Not all of the episodic memory data fit so easily into this interpretive scheme. Roediger and Tulving (1979), in some further experiments, reported one finding that fits with the previous analysis and one that does not. More specifically, they exposed subjects to lists of 64 words ( 8 words from each of 8 categories) and asked them to recall only those words not beginning with four particular letters. This instruction, which eliminated 32 words on the list, would not be expected to alter the size of the search set because, presumably, subjects did not encode the list items in terms of each word's first letter. Instead, subjects would be expected to search as they ordinarily would and simply ignore retrieved words beginning with the forbidden letters. Thus, relative to a control group instructed to recall all 64 words, the exclusion manipulation should decrease asymptotic recall by about $50 \%$ without affecting the rate of approach to the asymptote. This was exactly what Roediger and Tulving found. Equation 1 was fit to the relevant data estimated from their Figure 1, and the results showed that whereas the exclusion manip- ulation decreased $N$ from 21.0 to $9.1, \lambda$ was equal to 0.54 in both cases. Thus, as with Metlay et al. (1971), $N$ and $\lambda$ were not inversely correlated because, theoretically at least, $N$ did not provide an index of search set size in this case.

A second condition studied by Roediger and Tulving (1979), however, produced a far more surprising result that is less easily reconciled with the random search model. Using the same procedure described above (i.e., lists of 64 words from eight different categories), they asked subjects to avoid recalling items from four categories on the list and to instead recall items from the remaining four categories (the names of which were not provided). Presumably, the list items, which were presented in blocked fashion and introduced by the category name, were encoded with respect to their semantic category. Thus, perhaps subjects would be able to avoid wasting time searching the excluded categories and effectively halve the size of the search set (i.e., $N$ should decrease by $50 \%$ and $\lambda$ should double). Instead, the value of $\lambda$ changed only slightly (from 0.54 to 0.65 ) on the basis of the data estimated from their Figure 1. As noted by Roediger and Tulving, this curious result seems to suggest one of two possibilities: (1) for some reason, subjects performed an obligatory search through the excluded categories (or at least had difficulty not thinking about those category names), or (2) the very idea that subjects

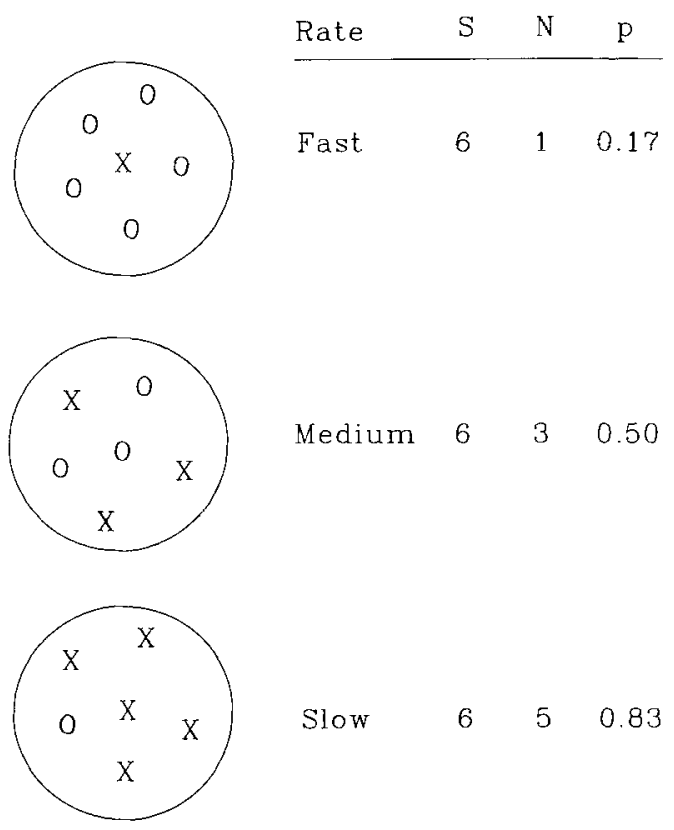

Figure 4. Hypothetical search sets established by six-item lists presented at fast, medium, and slow rates (top, middle, and bottom, respectively). $X$ represents an intact representation, whereas $O$ represents a degraded representation. $S=$ total items in search set, $N=$ number of intact items in search set, $p=$ probability that a list item is encoded in intact form. 
are searching through mental search sets is mistaken. As of yet, the issue has not been further pursued.

One way to shed some light on this interesting phenomenon might be to use an uninhibited recall procedure introduced by Bousfield and Rosner (1970) and used on occasion by others (e.g., Hogan, 1975; Roediger \& Payne, 1985). Subjects in these experiments were asked to verbalize any items that came to mind during the recall period even if they had been retrieved before. As might be expected, the same words were retrieved on multiple occasions. The use of this technique in conjunction with the exclusion procedure used by Roediger and Tulving (1979) could help indicate whether or not subjects actually spend time maladaptively retrieving items from (or simply thinking about) the excluded categories.

With the possible exception of this last result, the random search model provides a simple explanation for both the exponential form of retrieval and the inverse relationship between search set size and $\lambda$. However, the model seems to conflict with other well-known properties of retrieval. In particular, the generation of items from semantic memory is clearly not completely random but instead involves obvious clustering of related items. Subjects asked to name countries, for example, might begin by naming North American countries, followed by South American countries, followed by European countries, and so on. Does such evidence render the random search model invalid? Opinions on this point differ, but the position taken by Metlay et al. (1971) probably reflects the modal view:

The random search model is a simplified description of verbal recall. It assumes that every item in the search set has an equal chance of being selected for examination at any moment, and that the time spent making a response, i.e., motor time, is negligible. Neither of these assumptions is entirely true. It has been shown that the response probabilities of the names in a category are unequal and that the probabilities of successive responses in free recall of a memorized set of words are not independent. There is evidence for both nonrandomness and motor time in the present experiment, although these factors had little effect on the exponential distribution of recall times and on the dependence of relative response rate upon category size. (pp. 217-218)

Thus, according to this view, the evident nonrandomness of recall excludes sampling-with-replacement as a complete theory of memory, which has been obvious for many years (cf. Bousfield \& Sedgewick, 1944). However, this is not to say that the simpler theory is fundamentally wrong (i.e., contemporary theories often assume the sampling and resampling of search set items) or that the interpretation provided by the parameters of the best-fitting exponential are incorrect, as will be seen in the next section. The clustering of recalled items, which is basically relegated to error variance in the analyses discussed above, has actually been the focus of several studies concerned with the dynamics of retrieval from semantic and episodic memory. The next section reviews this research and considers its implications for the basic random search model in more detail.

\section{CLUSTERING}

A long and ongoing line of memory research has been concerned with the interesting fact that subjects tend to recall semantically related items together in time (e.g., Bousfield, 1953; Bousfield, Puff, \& Cowan, 1964; Deese, 1959; Pollio, 1964; Romney, Brewer, \& Batchelder, 1993; Schwartz \& Humphreys, 1973; Tulving, 1962). Most of this research has been concerned with whether or not adjacent items in a recall protocol appear together with a probability that is greater than would be expected on the basis of chance. A few studies have also investigated the hypothesis that interresponse times (IRTs) should be shorter for words within a semantic cluster than for adjacent words that form the boundary between two clusters.

\section{Semantic Memory}

Pollio (1964) was the first to study the relationship between semantic clustering and the dynamics of retrieval. In this experiment, subjects were asked to generate associations to a stimulus word (e.g., trouble). All responses were tape-recorded, so the experimenter had a verbatim record of responses as well as an accurate measure of when those responses occurred. As with retrieval from natural categories, the time course of recall on this task was approximated by Equation 1. The entire collection of IRTs (i.e., the times between consecutive retrievals) was divided into quartiles, and response clusters were identified by finding sequences of consecutive responses in which all of the IRTs fell within the fastest quartile. For example, if the three IRTs separating the words problem-fix-broken-mend all fell within the fastest quartile, but the IRTs preceding the word problem and following the word mend did not, then that four-word sequence would be identified as a cluster. Nonclusters were identified by finding sequences of responses in which all of the IRTs fell within the slowest quartile. Further analyses of the words comprising temporal clusters showed them to be more semantically related than words comprising nonclusters. For example, clustered items were more likely to be recalled together across individuals than were nonclustered items. Thus, related words not only appear together in recall protocols, which many previous studies have found to be true, they also occur as part of a burst of responding.

Graesser and Mandler (1978) performed a similar experiment in which subjects were asked to generate items from natural categories, but they used a different method for detecting clustering on the basis of temporal properties. The procedure was fairly involved and will only be briefly described here. First, a criterion IRT was selected arbitrarily (e.g., $3 \mathrm{sec}$ ), and potential clusters were identified by finding strings of successive responses with IRTs 
less than the critical value. For a given criterion IRT value, the mean size of all clusters identified in this way was calculated. This process was repeated for a range of criterion values, which allowed for a plot of mean cluster size versus criterion IRT. As the criterion IRT increased, mean cluster size increased in a negatively accelerated fashion up to a point (i.e., cluster size seemed to level off) and then in a positively accelerated fashion from that point on (i.e., cluster size began increasing rapidly again). The criterion IRT at the point of inflection was selected as the critical IRT, which was then used to identify clusters for further analysis.

Their analysis of within-cluster IRTs identified in this way revealed three important findings: (1) IRTs increased as a function of output position within a cluster (e.g., the second IRT of a cluster was longer than the first), (2) IRTs decreased as a function of cluster size at a given output position (e.g., the first IRT of a six-item cluster was shorter than that for a three-item cluster), and (3) cluster size had no effect on IRTs when they were plotted in backward serial order (e.g., the last IRT was the same regardless of cluster size). Concerning this last point, Graesser and Mandler (1978) state, "The critical determinant of the pattern of IRTs is how many items are left to be emitted from a cluster. When there are $n$ items left to be emitted from a cluster, the IRTs are the same for all cluster sizes" (p. 98). Although it is not intuitively obvious, these three findings are actually diagnostic of a constant probability search process, such as sampling-with-replacement, at the level of individual clusters.

To see why this is true, first consider the expected IRT output function assuming random search at the level of an entire list. That IRTs increase with successive retrievals according to the random search model is already established by the fact that Equation 1 implies that recall is a negatively accelerated process. Albert (1968, cited in Vorberg \& Ulrich, 1987) noted that constant probability search models predict that IRTs will grow according to $(1 / \lambda) /(N-i)$, where $i$ denotes output position (i.e., IRT $_{1}$ refers to the time separating the retrieval of the first and second items, IRT $\mathrm{IR}_{2}$ refers to the time separating the retrieval of the second and third items, etc.), and $N$ represents the number of items eventually retrieved. For the sampling-with-replacement model, which is one of the simplest constant probability search models, $\lambda$ in this equation can be replaced by $r / S$ (Equation 3), such that

$$
\mathrm{IRT}_{i}=\frac{S}{r(N-i)},
$$

where $N$ is now interpreted as the number of recoverable items in the search set (and still also represents asymptotic recall). Murdock and Okada (1970) found that IRTs in episodic memory experiments do increase in a positively accelerated fashion as predicted by Equation 4, although they did not provide a rigorous mathematical anal$y$ sis of the growth function.

The sampling-with-replacement model underlying Equation 4 assumes that a single search set is established by a retrieval cue (e.g., sports) and that items are randomly sampled from that set. Although this model is useful for addressing certain questions, the studies of clustering reviewed above suggest that a more accurate model must assume that nested search sets are established during the course of retrieval (e.g., outdoor sports, indoor sports, etc.). The identical random search model can be applied at the level of individual clusters, except that now $S$ refers to the size of a nested search set (e.g., indoor sports) and $N$ represents the number of target items in that set.

Graesser and Mandler's (1978) three main findings concerning within-cluster IRTs follow directly from Equation 4 (applied at the level of nested search sets) if $S$ is assumed to remain constant and cluster size is assumed to reflect the number of recoverable targets in a nested search set (i.e., $N=$ observed cluster size). Obviously, IRTs should increase with output position according to Equation 4 (Graesser and Mandler's first point). Furthermore, for a given search set size, Equation 4 also suggests that IRTs should decrease as cluster size increases (Point 2 above). To illustrate why this is so, consider two clusters, one of size $3(N=3)$ and the other of size 6 $(N=6)$. Assume, for the sake of illustration, that both clusters were drawn from hypothetical search sets of 9 total representations $(S=9)$. If one target item from each search set has already been retrieved, then the probability of successfully selecting a new target item on the next draw is $2 / 9$ in the first case and 5/9 in the second case. Thus, the time required to find a second target item will be significantly shorter in the latter case. Finally, Equation 4 predicts that IRTs will be the same regardless of cluster size when they are plotted in backward serial order (Point 3 above). Continuing with the previous example, if all but the last target item has been retrieved (i.e., 2 have been retrieved in the first case, 5 in the second), then the probability of selecting the last target item on the next draw is $1 / 9$ in both cases (because, in both cases, 1 target item remains to be found among 9 total items in the hypothetical search sets). Thus, the time between the secondto-last and last retrieval will be the same regardless of cluster size. Referring to Equation 4, for the last IRT the denominator is always equal to 1 regardless of the value of $N$ (thus, the size of the last IRT will be independent of the number of targets in the cluster-level search set). It should be noted that this analysis depends on the assumption that $S$ (nested search set size) is the same for each cluster, which means that some items in a nested search set are not recoverable.

To this point, the random search model has been applied at the level of lists as well as the level of clusters. Combining these two ideas suggests a hierarchical sampling-with-replacement scheme. Semantic fields (or subcategory names) comprise one level of search, and the items within each field comprise a nested level of search. According to this model, the time between successive subcategory retrievals should increase with output position (i.e., the time between the first and second clusters should be less than the time between the second and third clus- 
ters), as should the time between successive retrievals within a subcategory (i.e., the time between the first and second items within a cluster should be less than the time between the second and third items of a cluster). The expected increase in IRTs at both levels occurs for the same reason-namely, as more items are recalled, draws from the search set are increasingly likely to retrieve alreadysampled items.

Several two-stage search models roughly similar to that described above have been proposed. Gruenewald and Lockhead (1980), for example, proposed such a hierarchical search model, but they argued against the samplingwith-replacement scheme for two reasons. First, their cumulative recall curves were more accurately described by the hyperbola than by the exponential (cf. Fitzgerald, 1983). Second, in contrast to the results reported by Graesser and Mandler (1978) and replicated by Rubin and Olsen (1980), the IRTs within identified clusters did not seem to increase with output position. The differences on this point may reflect differences in the way in which clusters were identified. Gruenewald and Lockhead (1980) identified clusters by finding sequences of words with IRTs that were faster than expected on the basis of the best-fitting hyperbola. Nevertheless, Gruenewald and Lockhead did report that the time between successive clusters increased with output position as predicted by a two-stage sampling-with-replacement model.

Herrmann and Pearle (1981) challenged the position taken by Gruenewald and Lockhead (1980) on the grounds that the expected form of the retrieval curves according to a sampling-with-replacement model depends on one's assumptions about how a previously retrieved cluster is searched. Herrmann and Pearle showed that the expected cumulative recall function is exponential when the time taken to review a previously retrieved cluster is roughly the same as that required to review a new cluster. A more hyperbolic function is expected when the time taken to review a previously encountered cluster is less than that required to review a new cluster. An important feature of the account offered by Herrmann and Pearle is that the interpretation of $\lambda$ obtained from fitting Equation 1 (or Equation 2) to cumulative recall data does not differ from that provided by the simpler noncluster version of the random search model. That is, $\lambda$ is still inversely related to overall search set size (i.e., number of nested search sets times the number of items per nested search set) even though the individually recalled items are organized into clusters. Thus, it should not be assumed that the mere presence of clustering necessarily disqualifies Equation 1 or the theoretical interpretation of its free parameters.

For mathematical simplicity, Herrmann and Pearle's (1981) analysis assumed sampling-with-replacement at the level of clusters, but not at the level of individual items within clusters. Instead, within-cluster retrieval was assumed to proceed in linear fashion. This is probably a reasonable approximation because within-cluster IRTs are generally much faster than between-cluster IRTs, in part because subjects may switch to a new nested search be- fore IRTs become long. Nevertheless, the results of Graesser and Mandler (1978) seem to imply that withincluster search might be better modeled by an account that assumes sampling-with-replacement at that level as well. Herrmann and Pearle commented that the manner in which such a scheme might be formally incorporated into their mathematical analysis is unclear. Presumably, such a modification would not dramatically alter the interpretation of $\lambda$ offered by the model, but a definitive statement on this point will not be possible unless the mathematical details are eventually worked out.

\section{Episodic Memory}

As in the case of semantic memory, retrieval from episodic memory often involves clear evidence of clustering (e.g., Tulving, 1962). Once again, Pollio and his colleagues were the first to examine the temporal properties of these clusters in detail (Pollio, Kasschau, \& DeNise, 1968; Pollio, Richards, \& Lucas, 1969). Pollio et al. (1968) asked subjects to recall lists of 22 related words. One list, for example, consisted of items related to the word music. A 2-min recall period followed list presentation during which recall responses were tape recorded (to allow for accurate timing). Clusters were identified on the basis of temporal transition points. Anytime an IRT was less than one fifth of the preceding IRT or greater than five times the preceding IRT, the beginning or end of a cluster was marked, respectively. An analysis of clusters identified in this way showed them to be more semantically related (e.g., they were more likely to be recalled together across subjects) than words appearing in separate clusters. Thus, as in the case of semantic memory, related items are retrieved as part of a burst of responding.

Pollio et al. (1969) used a procedure that made clustering much more likely and the identification of response clusters much easier. Subjects were asked to memorize and then recall lists of 25 words composed of 5 words from each of 5 natural categories (e.g., countries, fruits, sports, etc.). If a subject recalled the words baseball, football, basketball, Canada, and Mexico (in that order), two response clusters would be plainly evident (one of size 3 , the other of size 2). As before, responses were taperecorded in order to facilitate an accurate temporal analysis. An analysis of the temporal properties of recall revealed basically the same pattern observed in the semantic memory experiments discussed above. That is, the results showed a progressive increase in IRTs as a function of output position within a cluster, as well as a progressive increase in IRTs between the end of one response cluster and the beginning of the next. In other words, as Pollio et al. point out, the results are consistent with a two-stage sampling-with-replacement search scheme.

Patterson, Meltzer, and Mandler (1971), using basically the same procedure as Pollio et al. (1969), replicated these findings (although within-cluster IRTs did not exhibit a clear increase with output position) and described the re- 
sults of a computer simulation of the two-stage random search model that has been repeatedly proposed over the years. In this model, category names were randomly sampled with replacement, as were items within a sampled category. As usual, items were overtly recalled the first time they were sampled and were simply ignored on subsequent retrievals. In addition, the model assumed that following each retrieval the subject evaluated the likelihood of finding another item in that category on the basis of the number of items that remained to be found. When that estimate was sufficiently low, the subject was assumed to return to the higher level search and randomly draw from the pool of categories, followed by another withincategory search. This model closely approximated the within-cluster and between-cluster IRT growth functions.

To briefly summarize, the simple random search model that generates Equation 1 and provides an interpretation of its free parameters may best apply under conditions that minimize clustering. Such conditions might involve the use of short lists of unrelated words or words that are drawn from a single semantic category. Under other conditions (e.g., large lists of words drawn from several semantic categories), a hierarchical search model with at least two leveis seems more appropriate. Nevertheless, the fundamental sampling scheme, which involves the sampling and resampling of search set items (which, in turn, results in a growing IRT function), need not be altered. Moreover, the mathematical analysis of cumulative recall by Equation 1 (or Equation 2) may help to quantify the scope of search through long-term memory, whether clustering is present or not.

\section{THE AMBIGUITY OF RECALL PROBABILITY}

The studies reviewed to this point were concerned with the relationship between the temporal properties of recall and the underlying search process. Beyond theoretical considerations such as these, another reason to study the time course of recall is to avoid interpretive ambiguities that might otherwise result. As indicated earlier, most list memory experiments report only one point on the cumulative recall curve-namely, the point reflecting progress subjects have made by the end of an arbitrarily defined recall period. Because recall is characterized by two properties, a single point estimate of performance can be ambiguous. For example, does $25 \%$ correct recall at the end of a 1-min recall period reflect a low level of asymptotic recall or is it merely one point on a slow path to $50 \%$ correct recall? In spite of this potential ambiguity, very few investigations of episodic memory have tracked its time course. Two exceptions are investigations into partlist cuing and hypermnesia.

\section{Output Interference and Part-List Cuing}

Slamecka $(1968,1969)$ reported a curious finding regarding the associative status of a memorized list of words. After studying a list, subjects in these experiments were presented with some of the items from the list to be used as retrieval cues for the remaining items. If the list items were associatively encoded, then one might expect these cues to facilitate access to the noncued items. Instead, Slamecka found that these items were actually less likely to be recalled relative to a control group that received no retrieval cues. This part-list cuing effect is similar to output interference effects observed in other contexts. For example, Brown (1968) found that subjects who were first asked to memorize and recall a list of 25 states were partially impaired in their ability to later generate all $\mathbf{5 0}$ states from semantic memory. More specifically, relative to a control group that was not pretested, the experimental subjects were less likely to generate states from the set of 25 that did not appear on the previously studied list.

Why does the presentation of part-list cues (or the prior recall of some subset of the list) serve to interfere with the recall of the remaining items? Roediger $(1974,1978)$ pointed out that the interpretation of these findings is complicated by the fact that recall may not be approaching asymptotic levels by the end of an arbitrary recall period. For example, in part-list cuing experiments, the rate of approach to asymptote could be reduced simply because of the extra time required to search through the cues after each retrieval to determine whether or not the retrieved item should be overtly recalled. If so, then the level of recall for the noncued items might be depressed merely because the recall period was too short to allow recall for those items to reach asymptotic levels.

Roediger, Stellon, and Tulving (1977) performed a detailed analysis of cumulative recall to determine whether part-list cues affect asymptotic recall or rate of approach to asymptote or both. The theoretical interpretation of the part-list cuing effect obviously depends on knowing which property of recall is affected. Subjects in various conditions studied a list of 48 words and were then given $10 \mathrm{~min}$ to recall those words. In one condition, subjects received 32 of the list items at the beginning of the recall period and were asked to recall the remaining 16 items. These 16 items were termed the critical items, and they were the same for all groups. Relative to a control group that attempted to recall all 48 items, both asymptotic recall and rate of approach to asymptote for the 16 critical items were reduced by the presentation of the 32 part-list cues. Thus, the effect of part-list cues is not entirely the result of terminating recall prematurely. Nevertheless, an adequate account of the phenomenon must explain why partlist cues affect both properties of recall.

As indicated above, the negative effect on rate of approach to asymptote could be caused by the extra checking operation associated with the 32 part-list cues. According to this idea, the subject first searches a mental search set, retrieves an item, and then searches an external search set (viz., the part-list cues) to determine whether or not the item should be overtly recalled. Because the effective size of the search set is increased, the rate of approach to asymptote should be correspondingly 
reduced (Equation 3). This hypothesis was evaluated by Roediger et al. (1977) by including another condition in which subjects were permitted to study the 32 part-list cues for 1 min prior to attempting recall of all 48 items on the list (during which time the cues were removed). Because the external search set is no longer present in this case, rate of approach to asymptote might be expected to match that of the control group. Instead, for the 16 critical items, rate of approach to asymptote was about the same as that for the part-list cued subjects. One possible interpretation of this result is that recall for the 16 critical items is delayed in both cuing conditions because the representations of the 32 part-list cues are strengthened such that they are sampled and retrieved first (whether the subject is asked to recall them or simply to use them as cues for the remaining items).

Rundus (1973) proposed a sampling-with-replacement model of retrieval consistent with this idea. His model assumed that each time an item is retrieved from a search set, its representation is strengthened such that it is more likely to be sampled again. Because not-yet-retrieved items are correspondingly less likely to be sampled in a given moment, the retrieval of those items will be delayed. Partlist cues may be functionally similar to previously retrieved items. That is, the cues are themselves strengthened such that they are more likely to be sampled. Note that this theory contrasts with the simplest exponential search scheme, which assumes a constant probability of sampling for items in the search set. Indeed, the presence of output interference is more easily reconciled with a hyperbolic growth function (cf. Bousfield et al., 1954).

If the part-list cues are strengthened such that they are preferentially sampled, this should affect rate of approach to asymptote while leaving asymptotic recall unaffected. Roediger et al. (1977), however, found that part-list cues reduce asymptotic recall as well. Why should that happen? Various explanations are possible. For example, if subjects employ a stopping rule, then delaying recall by any method will always reduce asymptotic recall as well (because subjects will give up before recalling otherwise accessible words). Another possibility is that the strengthening of some items by presenting them as cues interferes with other, noncued, items such that their status is changed from recoverable to nonrecoverable. At the present time, the available evidence does not allow a clear distinction between these possible explanations.

\section{Hypermnesia}

Hypermnesia refers to an increase in overall recall probability with successive recall tests following list presentation. Erdelyi and Becker (1974), for example, presented subjects with lists of 80 items, consisting of 40 nouns and 40 easily named pictures. Following list presentation, subjects were given three successive 7-min free recall tests. For the words, recall performance was essentially flat across the three tests; however, for pictures, performance actually increased with each test. This pattern (i.e., greater hypermnesia for pictures than for words) has been observed repeatedly.
The questions of interest in this line of research are (1) Why does hypermnesia occur? and (2) Why is it restricted to pictorial representations? The answers to these questions become apparent upon examination of the time course of free recall. Roediger and Thorpe (1978) observed that cumulative recall curves are often still increasing at the end of an arbitrarily defined recall period (cf. Figure 2). They reasoned that three successive 7-min recall periods may result in incremental improvement because subjects are, in effect, given $21 \mathrm{~min}$ to recall list items. Perhaps the extra recall time simply allows the cumulative recall curve to make further progress toward its final asymptote. If so, then a comparison of two groups, one given three successive 7-min recall periods and the other given a single 21-min recall period, should yield identical results.

Roediger and Thorpe (1978) performed this experiment using lists of 50 easily named pictures or 50 words. Half the subjects in each group received three 7-min recall tests, whereas the other half received a single 21-min recall test. Unlike in earlier experiments, these authors found evidence of hypermnesia for both pictures and words (for reasons that are unclear). More to the point, they found no differences in performance between subjects given three successive recall tests and those given a single 21min recall test. The cumulative recall curves for each condition are shown in Figure 5. Note that subjects in the successive testing condition recall many of the same items three times. For the plot shown in Figure 5, cumulative recall is incremented only when an item is recalled for the first time (which is, of course, also true of the subjects in the 21-min test condition).

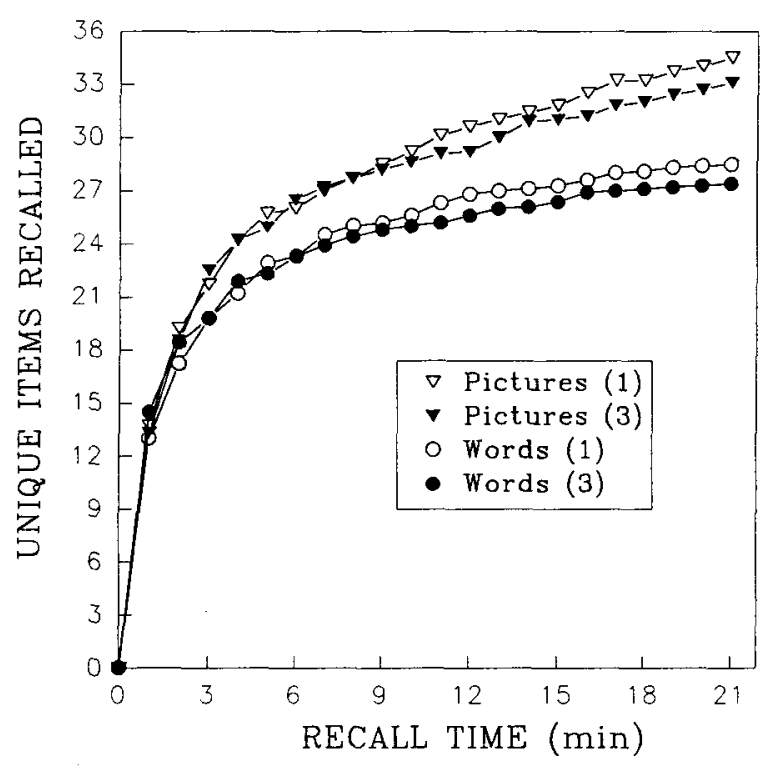

Figure 5. Cumulative recall functions produced by a single 21 min recall period versus three successive 7 -min recall periods for pictures and words. The data were taken from Roediger and Thorpe (1978). 
The analysis of cumulative recall curves also helps to address the second question pertaining to hypermnesia: Why is it more likely to occur for pictures than for words? One hypothesis is that hypermnesia depends on the imaginal encoding of list items. Erdelyi, Finkelstein, Herrell, Miller, and Thomas (1976), for example, presented subjects with a list of easily named pictures or the names of easily imagined objects. For subjects receiving the names of objects, some were instructed to form vivid mental images of those objects and some were not. Hypermnesia was clearly evident when subjects received pictures or were instructed to form mental images, but not when they were simply given a list of words to memorize. Thus, these results support the idea that imaginal encoding underlies hypermnesia.

Roediger, Payne, Gillespie, and Lean (1982) considered another possible explanation. They observed that manipulations that increase the likelihood of obtaining hypermnesia also increase the asymptotic level of recall. This is an important observation because much of the research reviewed earlier suggests that $\lambda$ (rate of approach to asymptote) is inversely related to $N$ (asymptotic recall). Thus, conditions that result in a higher level of asymptotic recall (e.g., pictures) are less likely to have reached the higher asymptote at the end of a 7-min recall period. Additional 7 -min recall periods would therefore allow for further progress (i.e., hypermnesia would be observed). By contrast, conditions that result in a lower level of recall (e.g., words) are more likely to have reached asymptote after $7 \mathrm{~min}$ because they are associated with a larger $\lambda$. In that case, additional 7-min recall periods will not be particularly helpful (i.e., hypermnesia will not be observed).

Actually, that hypermnesia for pictures generally exceeds that for words can be explained even without assuming that $N$ and $\lambda$ are inversely correlated. That is, the argument holds if the only difference between two conditions is in asymptotic recall. For example, assume that the cumulative recall function for pictures is $F(t)=30(1$ $\left.-\mathrm{e}^{-.25 t}\right)$, whereas that for words is $F(t)=20\left(1-\mathrm{e}^{-.25 t}\right)$, with $t$ measured in minutes. As calculated from these equations, the number of pictures recalled will be about 24.8 after $7 \mathrm{~min}$ and about 29.1 after another $7 \mathrm{~min}$. Thus, for two successive 7 -min tests, recall should increase by 4.3 items. For words, the number recalled will be about 16.5 after $7 \mathrm{~min}$ and about 19.4 after another $7 \mathrm{~min}$. Thus, for two successive 7-min tests, recall should increase by only 2.9 words. These calculations reveal that less hypermnesia is expected for lower asymptotic recall levels even if $\lambda$ is equivalent in the two conditions.

In any case, to test the idea that differences in asymptotic recall might underlie the picture-word effect, Roediger et al. (1982) presented subjects with a list of 60 words to memorize and used a standard manipulation to vary the number of words recalled. For 20 of these words, subjects were asked to verify whether or not a particular letter appeared in the word (a manipulation designed to induce graphemic processing). For another 20 words, subjects were asked whether or not the list word rhymed with a test word (phonemic processing). For the remaining 20 words, they were asked whether or not the word was a member of a category (semantic processing). Half the subjects in each group received three 7 -min recall tests in succession, and the other half received a single 21-min recall test. As expected, semantic processing resulted in a higher level of asymptotic recall than phonemic processing, which, in turn, yielded a higher level of asymptotic recall than graphemic processing. Thus, additional 7-min tests should yield greater absolute gains for the semantically processed items. Indeed, hypermnesia (the improvement in recall across successive 7-min tests) was greater for semantically processed words than for words that received either phonemic or graphemic processing.

Experiments performed subsequently have suggested that differences in asymptotic recall may not fully explain differences in hypermnesia for pictures versus words. Payne (1986), for example, presented lists of 60 words at a fast or slow rate and lists of 60 easily named pictures at a fast or slow rate. For the slowly presented words and rapidly presented pictures, asymptotic recall was essentially equal. Nevertheless, hypermnesia was still greater for pictures than for words. The reason for the difference is that words suffered a greater amount of intertest forgetting than did pictures when three successive 7-min recall tests were used.

These considerations reveal that the mathematical analysis of long-duration cumulative recall curves might be somewhat tricky. When relatively short recall periods are used, the amount of information lost during recall due to forgetting is probably negligible, but a lot of forgetting can occur over $21 \mathrm{~min}$. Thus, to perform a search set analysis based on Equation 1, $\lambda$ would need to be disentangled from a forgetting rate parameter (which may differ across conditions).

Previous research has shown that the course of forgetting is usually well described by a power function of the form $a t^{-b}$ (Anderson \& Schooler, 1991; Wixted \& Ebbesen, 1991). Can exponential retrieval be integrated with power function forgetting? The random search model assumes that the rate of recall during the recall period is equal to the rate of sampling $(r)$ times the probability of sampling a new target item on a given draw, which is equal to $(N-n) / S$, where $n$ is the number of target items sampled thus far (so $n$ eventually equals $N$ ). If no forgetting is assumed, then the solution to this differential equation yields Equation 1, the exponential cumulative recall curve (McGill, 1963). However, the assumption of no forgetting becomes increasingly untenable as the recall period becomes longer. A more realistic model would assume that the rate of recall is equal to the rate of sampling, $r$, times the probability that a new target representation is sampled, $(N-n) / S$, times the probability that the target will be recovered when sampled. The latter probability can be assumed to decline as a function of time, according to a power function of the form $(t+1)^{-\alpha}$. 
Written this way, the power function begins at 1.0 when $t=0$ (i.e., no encoded targets yet forgotten) and decreases to 0 as $t$ approaches $\infty$ (i.e., all target items forgotten). The solution to this new differential equation (details given in the Appendix) yields the following equation:

$$
F(t)=N\left(1-\mathrm{e}^{(\lambda / \beta)\left(1-(t+1)^{\beta}\right]}\right),
$$

where $\beta$ is the forgetting function parameter that ranges from a maximum of 1.0 (no forgetting) down to minus infinity (instantaneous forgetting). Note that when no forgetting occurs (i.e., $\beta=1$ ), Equation 5 reduces to Equation 1 , as it should. Furthermore, when $\beta$ is between 0 and 1, recall will be slowed, but all $N$ recoverable items will be sampled before they become unrecoverable (i.e., $N$ = asymptotic recall as before). However, when $\beta$ is less than 0 (as it is in the example to be considered below), some items that were theoretically encoded in recoverable form will become unrecoverable before they are sampled. Thus, in this case, $N$ only represents the number of targets initially encoded, not asymptotic recall. To better illustrate this point, Equation 5 can also be written as

$$
F(t)=N-k(N / k)^{(t+1)^{\beta}},
$$

where $k$, which is equal to $N \exp (\lambda / \beta)$, represents the number of targets forgotten before they can be retrieved (assuming $\beta$ is negative). Thus, asymptotic recall in this case is equal to $N-k$. Equations 5 and 6 are actually the same equation written in different forms. Both are three-parameter equations, but the inclusion of a forgetting rate parameter seems inescapable when recall periods become very long.

How well do these equations describe episodic cumulative recall data when long recall periods are used? Figure 6 shows the Roediger and Thorpe (1978) data presented in Figure 5, except that the data from the two recall test conditions $(3 \times 7$-min vs. 21 -min recall $)$ were averaged together for both pictures and words. The upper panel shows the best fit provided by Equation 1, and it clearly reveals that the fit is inadequate. The lower panel shows the fit provided by Equation 6, which is obviously much better.

Is the improved fit nothing more than yet another demonstration that the addition of free parameters always helps? Actually, several arguments suggest that the improved fit may be more than that. First, the choice of the power forgetting function was not arbitrary but was instead based on existing information about the typical form of forgetting. When analyzing forgetting functions, the exponential generally provides a poor fit whereas the power function generally provides a much better fit (Anderson \& Schooler, 1991; Wixted \& Ebbesen, 1991). When Equations 5 and 6 are derived assuming exponential forgetting (which also adds a third free parameter), the resulting equations continue to exhibit large and systematic deviations from the data (although some improvement occurs because of the extra parameter). Visually, the fit does not look much different from that provided by the simple exponential. This is what would be expected

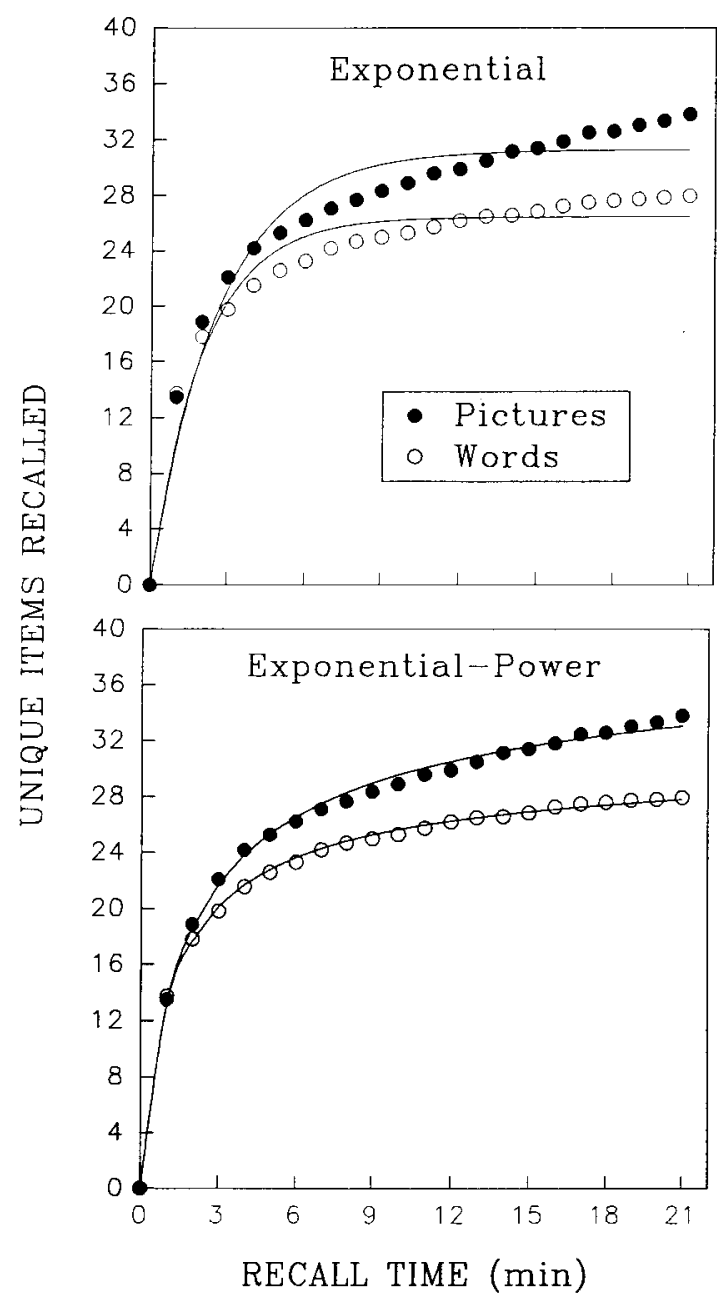

Figure 6. Upper panel: Cumulative recall functions for pictures and words (averaged over the $3 \times 7$-min and 21-min recall conditions). The solid curves represent the least squares fit of Equation 1. Lower panel: The same data, with the solid curves representing the least squares fit of Equation 6. The data were taken from Roediger and Thorpe (1978).

if the present analysis were on the right track, because exponential forgetting is an incorrect assumption.

Second, the values assumed by the free parameters, which are presented in Table 2, turn out to be sensible. For the words, the parameters obtained from Equation 6 suggest that 40.9 of the 50 items were initially encoded, but 7.8 were lost before they could be retrieved. Thus, asymptotic recall in this case would be equal to 40.9 7.8, which equals 33.1. For the pictures, the parameters suggest that 48.8 items were initially encoded, but 3.6 were forgotten before they could be retrieved. Asymptotic recall in the case would be $48.8-3.6$, which equals 45.2. Thus, the estimated number of items forgotten during recall seems reasonable.

Third, the rate of forgetting is estimated to be less for pictures than for words $(-.19$ vs. -.38$)$. As indicated 
Table 2

Parameter Values Obtained From Fitting Equation 6 to the Cumulative Recall Curves Shown in Figure 6

\begin{tabular}{lccc}
\hline & $N$ & $k$ & $\beta$ \\
\hline Words & 40.9 & 7.8 & -.38 \\
Pictures & 48.8 & 3.6 & -.19 \\
\hline
\end{tabular}

above, when three successive recall periods are used, some items recalled on earlier tests are forgotten on later tests. Thus, if we assume that the forgetting of not-yetretrieved items is similar to the forgetting of alreadyretrieved items, then the relative amount of between-test forgetting in a hypermnesia experiment can be used to assess forgetting rate differences. Roediger and Thorpe (1978) found no differences between pictures and words on this measure, whereas Payne (1986), using similar materials, found that forgetting was less for pictures than for words. Given the inconsistent findings (and, more importantly, the absence of actual forgetting functions), it is difficult to evaluate whether or not the estimated forgetting rate difference between pictures and words is accurate. Nevertheless, Payne's (1986) data at least hint that pictures are less likely to be forgotten than words, as the present analysis suggests.

Note that nothing in the data shown in Figure 6 suggests that any forgetting occurred, much less that the rate of forgetting was less for pictures than for words. Nevertheless, the use of Equation 6 suggests that the degree of deviation from the expected exponential course of retrieval implies that forgetting did indeed occur and that it was less for pictures than for words.

Roediger and his colleagues have repeatedly observed that the failure to track cumulative recall can lead one to severely underestimate the number of items that can be recalled. For the data shown in Figure 6, for example, had recall been terminated after 3 min (which is longer than most studies allow), the number of pictures recalled would have been about 22. Visual inspection of Figure 6 suggests that asymptotic recall would actually be well above 36 items, and the use of Equation 6 suggests that asymptotic recall would be closer to 45 items (although a very long recall period would be required). Note that even asymptotic recall may not be the best measure of performance when the rates of forgetting differ between conditions. Instead, $N$ (the estimated number of items initially encoded) might be the preferred dependent measure. For example, if two conditions result in the initial encoding of the same number of items, but the rate of forgetting in one condition exceeds that in the other, then asymptotic recall will differ in the two conditions. That difference merely reflects forgetting rate differences, not differences in the amount of material learned.

\section{ALTERNATIVE THEORETICAL MODELS}

Throughout this review, a basic random search model was used to interpret and link together various investiga- tions into the time course of free recall. The simplicity of this model allows it to serve that conceptual role, but also disqualifies it from serving as a comprehensive theory of retrieval. Conversely, more complex accounts can serve as comprehensive theories, but are less successful in helping to make sense of seemingly disparate findings. Nevertheless, the question of whether or not such theories can accommodate the relevant findings is a reasonable one to ask. In this section, some alternatives to the random search model are considered, beginning with its translation into a parallel search model.

\section{Parallel Versus Serial Processing}

The random search model is generally described in serial terms. That is, an item is assumed to be randomly selected from a search set, evaluated, and then replaced, after which the next item is randomly sampled, evaluated, and replaced, and so on. However, the search for encoded list items may not be sequential in nature. According to the parallel search model, items in a search set are simultaneously activated and each has the same momentary probability of reaching conscious awareness following activation (e.g., McGill, 1963; Vorberg \& Ulrich, 1987). That some items become conscious almost instantly whereas others require a much longer period of time is attributable to chance. Moreover, according to this model, cumulative recall will be described by Equation 1 and $\lambda$ reflects the momentary probability of retrieval associated with individual items. Thus, for example, if each activated item has a constant per-second probability of reaching awareness equal to .25 , then $\lambda$ will equal .25 (and average recall latency will equal $4 \mathrm{sec}$ ).

It may not be possible to distinguish between serial and parallel models in practice, as Vorberg and Ulrich (1987) have pointed out, but certain results seem to fit more naturally into one model or the other. For example, parallel processing in visual search is suggested by the fact that, under some conditions, the number of distractors in a visual display does not affect the time taken to find a target (e.g., Treisman, 1986). Presumably, adding distractors merely increases the number of simultaneous searches. Similarly, if enlarging a mental search set by adding additional targets or distractors failed to affect $\lambda$, that result would be most easily interpreted in terms of a parallel model. For example, if $S=5$ (i.e., the activated search set contains 5 items) and the momentary probability of retrieval associated with each of those 5 items was .10, then $\lambda$ would equal .10. If 5 additional target items were added to that search set, each with a .10 momentary probability of retrieval, then $\lambda$ would remain equal to .10 (though asymptotic recall would double).

The simplest serial model, by contrast, predicts that $\lambda$ will decrease as $S$ increases according to Equation 3. Most of the literature reviewed earlier seems to suggest that $\lambda$ is indeed inversely related to $S$, as the serial model suggests. Thus, for example, when subjects are asked to generate items from a semantic category, $\lambda$ decreases as 
category size increases. Similarly, as episodic list length increases, or as PI builds (both of which theoretically increase search set size), $\lambda$ decreases. Results such as these are most easily interpreted in terms of a serial model that suggests an inverse relation between $S$ and $\lambda$. Nevertheless, they do not rule out the parallel version. For example, if one assumes a finite pool of activation that is spread evenly over all $S$ items, then the interpretations provided by the parallel and serial models become identical (i.e., $S$ and $\lambda$ should be inversely related).

\section{Mathematical Modifications of Random Search}

Several assumptions of the random search model are, at best, approximations to the truth. In episodic memory experiments, for example, the model assumes that no forgetting occurs during the course of recall. Although this assumption may be essentially correct when a short recall period is used, it becomes increasingly untenable as the length of the recall period increases. In addition, the simplest version assumes that (1) clustering does not occur because items are independently sampled, (2) each item in the search set is as likely as any other to be sampled on a given draw, and (3) the dynamics of retrieval are the same for every subject in the experiment. None of these assumptions is true, and the previous analysis assumed that violations of them do not alter the general interpretation offered by the random search model. Nevertheless, in some cases (e.g., if clustering is expected to differ substantially across conditions), it may be preferable to apply a more exact mathematical analysis that addresses one or more of these potential problems.

Three articles appearing in the Journal of Mathematical Psychology have dealt with the problem of relaxing one of these assumptions (Herrmann \& Pearle, 1981; Morrison, 1979; Vorberg \& Ulrich, 1987). These articles present very detailed mathematical treatments that will only be touched on here. As already discussed, Herrmann and Pearle (1981) addressed the mathematics of clustering and showed that a random sampling model will yield an exponential or a hyperbolic cumulative recall curve (or something in between) depending on how clusters are treated when they are sampled again after already having been retrieved once. Morrison (1979) ignored the problem of clustering and instead considered how to deal with the problem of individual differences in rate of approach to asymptote $(\lambda)$. He showed that when exponential functions with different values of $\lambda$ are averaged together, the best-fitting exponential to the aggregate cumulative recall curve will initially overestimate and subsequently underestimate the level of recall. Indeed, when the exponential fails to provide a good fit, this is the usual way in which it deviates from the data. Vorberg and Ulrich (1987) ignored the problem of clustering and the problem of individual differences and instead concentrated on the effect of relaxing the assumption that each item in a search set has an equal probability of being sampled. When subjects are asked to generate pets, for example, the words dog or cat are more likely to be sampled than is the word hamster. Again, they showed that the bestfitting exponential to the cumulative recall curve will initially overestimate and subsequently underestimate the level of recall (the usual deviation) when items have different probabilities of being sampled.

These modifications to the random search model do not attempt to cope with all of the nuances of retrieval. For example, none deals with the effect of output interference or changes in the subject's willingness to continue searching for items during the course of recall. Other models designed to accommodate such effects have been developed in recent years.

\section{Search of Associative Memory}

The search of associative memory (SAM) model of human memory was initially conceived as a more realistic extension of the random search model (Shiffrin, 1970). Indeed, it still shares many features with the random search account, so it is perhaps not surprising that SAM provides an adequate account of cumulative recall. Very briefly, SAM assumes that a retrieval cue serves to activate a search set consisting of a collection of "images" (i.e., units of memory that contain the desired item). Some images are more strongly activated than others because they are more strongly associated with the retrieval cue. Once activated, the probability that an individual image will be sampled depends on its relative strength. Once sampled, the desired list item may or may not be recovered. That is, it may not be possible to reconstruct the original list item from the image it created. The same idea was alluded to earlier when considering the effect of study time on rate of approach to asymptote (cf. Figure 4).

Note that if every image in the search set were activated to the same degree, and this remained true throughout the recall period, then the sampling of images would be tantamount to a random search. However, other components of SAM serve to differentiate it from the vastly simpler random search model. For example, once retrieved, the item is not only replaced but its association with the retrieval cue is strengthened such that it is more likely to be sampled again. In addition, each retrieved item is assumed to act as a new retrieval cue that serves to activate and sample other items. Finally, subjects are assumed to employ a "stopping rule" if a long period of time elapses without a successful recovery.

In spite of these elaborations, it seems that under most conditions SAM provides essentially the same interpretation of $\lambda$ that the random search model provides. Thus, for example, as list length increases, the number of relevant images increases (i.e., search set size increases), which decreases the relative activation associated with any one image. Therefore, $\lambda$ should decrease. Indeed, simulations provided by Raaijmakers and Shiffrin (1980) show this to be true of SAM. They did not actually provide a mathematical analysis of their simulated cumulative recall curves, but they noted that they appear exponential in form. When Equation 1 is fit to their simulated data (estimated from their Figure 11), it provides an excellent 
fit and $\lambda$ decreases as simulated list length increases from 10 to $\mathbf{4 0}$ items. For the 10 -item list (1-sec presentation rate), the best-fitting exponential was $3.9\left(1-e^{(x) 7 t}\right)$. whereas the corresponding equation for the 40 -item list was $9.5\left(1-e^{-.038 t}\right)$.

Although SAM provides a reasonable account of cumulative recall (e.g., Gronlund \& Shiffrin, 1986), it should be clear that, unlike the random search model, its interpretation of $\lambda$ is not necessarily fixed. That is, if an experimental manipulation results in a decrease in $\lambda$, the random search model is bound by Equation 2 to assume that search set size increased (or that the sampling rate decreased). SAM, by contrast, can explain such results in a variety of ways.

\section{Distributed Memory Models}

Another aspect of the random search model that almost surely represents an oversimplified view of memory is the idea that items are stored as unitary traces in a physically localized search set. For some time now, the available physiological evidence has suggested that memory is probably better thought of as distributed (e.g., Lashley, 1950). Although the random search model could be described in distributed terms, an altogether different distributed memory model designed to explain free recall was proposed by Metcalfe and Murdock (1981). This model is one of a series of distributed memory models developed by these authors in recent years (e.g., Eich, 1982; Murdock, 1982, 1983). The central idea is that individual item representations are combined (by means of convolution) into a single memory vector $(\mathbf{M})$ instead of being stored as separate entities in a search set. Thus, once convolved, individual items share the same neural substrate. A retrieval cue extracts an individual item's representation from $\mathbf{M}$ by the operation of correlation instead of by random sampling.

Convolution and correlation are mathematical operations analogous to addition and subtraction. For example, assume that item $A$ can be represented along three semantic dimensions by the numbers $.5,-.4$, and .6 , whereas item $B$ can be represented along those same dimensions by the numbers $.2, .1$, and -.2 . Adding these vectors yields a combined vector (analogous to a convolved trace) of $.7,-.3$, and .4. This grand vector, denoted here by $\mathbf{M}$, contains the information associated with both items, although it now resembles neither of them. The re-presentation of item $B$ can be used as a cue to retrieve item A by simple subtraction of item B's vector from the grand vector, an operation analogous to correlation.

In the model described by Metcalfe and Murdock (1981), free recall of a recently presented list of words occurred in the following way. First, at the recall signal, the last rehearsed item was correlated with $\mathbf{M}$, which served to retrieve another item's representation. That representation was then correlated with $\mathbf{M}$, which retrieved another item's representation, which in turn was correlated with $\mathbf{M}$ and so on. This process continued until a certain amount of time elapsed without a successful retrieval ( $3 \mathrm{sec}$ in the simulations they reported), at which point the representation of the encoding context was correlated with $\mathbf{M}$. That operation may retrieve a new item, which may then be used as a retrieval cue for another item, and so on, until another search failure occurs.

This model, unlike SAM, is based on a view of retrieval fundamentally different from that of the random search model. Nevertheless, certain aspects of the two accounts are analogous. The convolved trace, $\mathbf{M}$, is analogous to a search set (i.e., it is the memory structure that contains the item representations). Furthermore, in both models, a serial retrieval operation is performed on the memory structure that leaves it unaltered, representations may or may not be sufficiently intact to reconstruct the original list item, and, as recall proceeds, more time will be required to find a not-yet-recalled item because of the reretrieval of previously recalled items. These similarities suggest that Metcalfe and Murdock's (1981) encoding and retrieval model might be able to provide a reasonable account of the dynamics of free recall and, in some cases at least, may provide an interpretation of $\lambda$ that is similar to that offered by the random search account.

At the present time, these are only speculations. The encoding and retrieval model as described by Metcalfe and Murdock (1981) was consistent with a large number of empirical free recall phenomena, but the timing of free recall was not among them. In particular, the model predicted an essentially flat IRT function, whereas in reality IRTs increase with output position (Equation 4). The probable reason for this odd prediction was the choice of a 3-sec stopping rule used throughout their simulations. The authors note that the use of a longer stopping rule produces an IRT growth function that is at least qualitatively similar to empirical IRT data.

\section{CONCLUSION}

The time course of free recall is an orderly phenomenon that is characterized by two properties: asymptotic recall and rate of approach to asymptote. When deciding upon the length of a recall period, researchers presumably allot what they believe to be enough time to allow performance to reach asymptotic levels. However, it seems likely that they are often mistaken in their assessment of the dynamics of free recall. Roediger and his colleagues have shown repeatedly that for long lists of words (e.g., 30 or more), recall may still be noticeably increasing even after $21 \mathrm{~min}$, which is far longer than the typical recall period. If recall is still progressing at the end of a recall period, then the percentage of correct responses at that point can be a misleading dependent measure. Indeed, failure to track the time course of recall led some to argue that hypermnesia was essentially "unforgetting." The relative lack of interest in cumulative recall is surprising in light of considerations such as these.

In addition to helping to resolve empirical ambiguities, the time course of recall may also provide unique insight 
into the nature of retrieval. Retrieval from both episodic and semantic memory follows a negatively accelerated time course that is described well by the exponential or the hyperbola. Much of the research reviewed earlier is consistent with the idea that the rate of approach to asymptote reflects the breadth of search undertaken to find the desired items. Indeed, that is the central theoretical conclusion to be drawn from this literature. Note that no new theory was needed to advance this hypothesis. The exponential form of retrieval and its connection to random search model have been known for decades. By its very nature, the random search model implies that the rate of approach to asymptote should be indirectly related to the size of the search set. Although the issue has rarely been explicitly addressed, the half century of research performed since Bousfield and Sedgewick's (1944) seminal work suggests that this implication may be correct. Furthermore, because it is not always clear how an experimental manipulation will affect the parameters of search, analyzing the dynamics of recall (in particular, rate of approach to asymptote) may turn out to be a profitable endeavor.

\section{REFERENCES}

Anderson, J. R., \& Schooler, L. J. (1991). Reflections of the environment in memory. Psychological Science, 2, 396-408.

BADDeley, A. (1990). Human memory: Theory and practice. Boston: Allyn and Bacon.

Bousfield, W. A. (1953). The occurrence of clustering in the recall of randomly arranged associates. Journal of General Psychology, 49 , 229-249.

Bousfield, W. A., Puff, C. R., \& Cowan, T. M. (1964). The development of constancies in sequential organization during repeated free recall. Journal of Verbal Learning \& Verbal Behavior, 3, 489-495.

Bousfield, W. A., \& RosNer, S. R. (1970). Free versus uninhibited recall. Psychonomic Science, 20, 75-76.

Bousfield, W. A., \& Sedgewick, C. H. W. (1944). An analysis of restricted associative responses. Journal of General Psychology, 30 , 149-165.

Bousfield, W. A., Sedgewick, C. H. W., \& Cohen, B. H. (1954). Certain temporal characteristics of the recall of verbal associates. American Journal of Psychology, 67, 111-118.

Brown, J. (1968). Reciprocal facilitation and impairment in free recall. Psychonomic Science, 10, 41-42.

Crowder, R. G. (1976). Principles of learning and memory. Hillsdale, NJ: Erlbaum.

DeESE, J. (1959). Influence of inter-item associative strength upon immediate free recall. Psychological Reports, 5, 305-312.

EICH, J. M. (1982). A composite holographic associative recall model. Psychological Review, 89, 627-661.

ERDELYI, M. H., \& BECKER, J. (1974). Hypermnesia for pictures: Incremental memory for pictures but not words in multiple recall trials Cognitive Psychology, 6, 159-171.

Erdelyi, M. H., Finkelstein, S., Herrell, N., Miller, B., \& THOMAs, J. (1976). Coding modality vs. input modality in hypermnesia: Is a rose a rose a rose? Cognition, 4, 311-319.

FitzGerald, J. M. (1983). A developmental study of recall from natural categories. Developmental Psychology, 19, 9-14.

Graesser, A., \& MANDLER, G. (1978). Limited processing capacity constrains the storage of unrelated sets of words and retrieval from natural categories. Journal of Experimental Psychology: Human Learning \& Memory, 4, 86-100.

Gronlund, S. D., \& Shiffrin, R. M. (1986). Retrieval strategies in recall of natural categories and categorized lists. Journal of Experimental Psychology, 12, 550-561.
Gruenewald, P. J., \& LockHead, G. R. (1980). The free recall of category examples. Journal of Experimental Psychology: Human Learning \& Memory, 6, 225-240.

Herrmann, D. J., \& Chaffin, R. J. S. (1976). Number of available associations and rate of association for categories in semantic memory. Journal of General Psychology, 95, 227-231.

HerrmanN, D. J., \& MurRay, D. J. (1979). The role of category size in continuous recall from semantic memory. Journal of General Psychology, 101, 205-218.

Herrmann, D. J., \& PEARLE, P. M. (1981). The proper role of clusters in mathematical models of continuous recall. Journal of Mathematical Psychology, 24, 139-162.

HoGAN, R. M. (1975). Inter-item encoding and directed search in free recall. Memory \& Cognition, 3, 197-209.

INDOW, T., \& TOGANo, K. (1970). On retrieving sequence from longterm memory. Psychological Review, 77, 317-331.

Johnson, D. M., Johnson, R. C., \& MARK, A. L. (1951). A mathematical analysis of verbal fluency. Journal of General Psychology, 44, 121-128.

Kaplan, I. T., Carvellas, T., \& Metlay, W. (1969). Searching for words in letter sets of varying size. Journal of Experimental Psychology, 82, 377-380.

LASHLEY, K. S. (1950). In search of the engram. Symposia of the Society for Experimental Biology, 4, 454-482.

McGILL, W. J. (1963). Stochastic latency mechanisms. In R. D. Luce, R. R. Bush, \& E. Galanter (Eds.), Handbook of mathematical psychology (Vol. 1, pp. 309-360). New York: Wiley.

MetCalfe, J., \& MURDoCK, B. B. (1981). An encoding and retrieval model of single-trial free recall. Journal of Verbal Learning \& Verbal Behavior, 20, 161-189.

Metlay, W., Handley, A., \& Kaplan, I. T. (1971). Memory search through categories of varying size. Journal of Experimental Psychology, 91, 215-219.

MoRRIson, D. G. (1979). An individual differences pure extinction process. Journal of Mathematical Psychology, 19, 307-315.

MuRDOCK, B. B. (1982). A theory for the storage and retrieval of item and associative information. Psychological Review, 89, 609-626.

MuRDOCK, B. B. (1983). A distributed memory model for serial-order information. Psychological Review, 90, 316-338.

Murdock, B. B., \& OKADA, R. (1970). Interresponse times in singletrial free recall. Journal of Experimental Psychology, 86, 263-267.

Patterson, K. E., Meltzer, R. H., \& Mandler, G. (1971). Interresponse times in categorized free recall. Journal of Verbal Learning \& Verbal Behavior, 10, 417-426.

PaYNe, D. G. (1986). Hypermnesia for pictures and words: Testing the recall level hypothesis. Joumal of Experimental Psychology: Learning, Memory, \& Cognition, 12, 16-29.

Pollio, H. R. (1964). Composition of associative clusters. Journal of Experimental Psychology, 67, 199-208.

Pollio, H. R., Kasschau, R. A., \& DeNise, H. E. (1968). Associative structure and the temporal characteristics of free recall. Journal of Experimental Psychology, 76, 190-197.

Pollio, H. R., Richards, S., \& LuCAS, R. (1969). Temporal properties of category recall. Journal of Verbal Learning \& Verbal Behavior, $8,529-536$.

RaAijmakers, J. G. W., \& Shiffrin, R. M. (1980). SAM: A theory of probabilistic search of associative memory. In G. H. Bower (Ed.), The psychology of learning and motivation: Advances in research and theory (Vol. 14, pp. 207-262). New York: Academic Press.

RoEDIGER, H. L., III (1974). Inhibiting effects of recall. Memory \& Cognition, 2, 261-269.

RoEdiger, H. L., III (1978). Recall as a self-limiting process. Memory \& Cognition, 6, 54-63.

Roediger, H. L., III, \& PAyne, D. G. (1985). Response criteria do not affect recall level or hypermnesia: A puzzle for generate-recognize theories. Memory \& Cognition, 13, 1-7.

Roediger, H. L., III, Payne, D. G., Gillespie, G. L., \& Lean, D. (1982). Hypermnesia as determined by level of recall. Journal of Verbal Learning \& Verbal Behavior, 21, 635-655.

Roediger, H. L., III, STELlon, C. C., \& TUlving, E. (1977). Inhibition from part-list cues and rate of recall. Journal of Experimental Psychology: Human Learning \& Memory, 3, 174-188. 
Roediger, H. L., III, \& Thorpe, L. A. (1978). The role of recall time in producing hypermnesia. Memory \& Cognition, 6, 296-305.

RoEDiger, H. L., III, \& TULVING, E. (1979). Exclusion of learned material from recall as a postretrieval operation. Joumal of Verbal Leaming \& Verbal Behavior, 18, 601-615.

ROHRER, D., \& WIXTED, J. T. (in press). An analysis of latency and interresponse time in free recall. Memory \& Cognition.

Romney, A. K., BreWer, D. D., \& Batchelder, W. H. (1993). Predicting clustering from semantic structure. Psychological Science, 4, 28-34.

Rubin, D. C., \& Olson, M. J. (1980). Recall of semantic domains. Memory \& Cognition, 8, 354-366.

Rundus, D. (1973). Negative effects of using list items as recall cues. Journal of Verbal Learning \& Verbal Behavior, 12, 43-50.

SCHWARTZ, R. M., \& HuMPHREYs, M. S. (1973). Similarity judgments and free recall of unrelated words. Journal of Experimental Psychology, 101, 10-15.

SHIFFrin, R. M. (1970). Memory search. In D. A. Norman (Ed.), Models of human memory (pp. 375-447). New York: Academic Press.

SlameCKA, N. J. (1968). An examination of trace storage in free recall. Journal of Experimental Psychology, 76, 504-513.

SLAMECKA, N. J. (1969). Testing for associative storage in multitrial free recall. Journal of Experimental Psychology, 81, 557-560.

Treisman, A. (1986). Properties, parts, and objects. In K. R. Boff, L. Kaufman, \& J. P. Thomas (Eds.), Handbook of perception and human performance: Vol. 2. Cognitive processes and performance (pp. 35-1 to 35-70). New York: Wiley.

TuLVING, E. (1962). Subjective organization in free recall of "unrelated" words. Psychological Review, 69, 344-354.

VoRBERG, D., \& ULRICH, R. (1987). Random search with unequal search rates: Serial and parallel generalizations of McGill's model. Journal of Mathematical Psychology, 31, 1-23.

WATKINS, O. C., \& WATKINS, M. J. (1975). Build-up of proactive inhibition as a cue-overload effect. Journal of Experimental Psychology: Human Learning \& Memory, 104, 442-452.

WiXted, J. T., \& EbBesen, E. (1991). On the form of forgetting. Psychological Science, 2, 409-415.

Wixted, J. T., \& RoHrer, D. (1993). Proactive interference and the dynamics of free recall. Journal of Experimental Psychology: Learning, Memory, \& Cognition, 19, 1024-1039.

\section{APPENDIX}

The differential equation that yields exponential retrieval (Indow \& Togano, 1970) is

$$
d n / d t=\lambda(N-n),
$$

where $n$ is the number of targets recalled by time $t$ and $\lambda$ is theoretically equal to $r / S$. Thus, for conceptual clarity, the equation may be rewritten as

$$
d n / d t=r\{(N-n) / S\} .
$$

This equation, which uses a continuous approximation of the discrete sampling process, states that the rate of recall, $d n / d t$, is equal to the rate of sampling, $r$, times the probability of sampling a new target item, $(N-n) / S$. When solved, this differential equation yields Equation 1. Here, we simply add the assump- tion that a sampled target is recognized as a list item with decreasing probability according to a power function such that

$$
d n / d t=r\{(N-n) / S\}(t+1)^{-\alpha} .
$$

This equation states that the rate of recall, $d n / d t$, is equal to the rate of sampling, $r$, times the probability of sampling a new target item, $(N-n) / S$, times the probability that the sampled target is recognized as a target, $(t+1)^{-\alpha}$. The latter value begins at 1.0 and declines to zero as $t$ approaches infinity. This new differential equation can be solved by first rearranging to

$$
d n /(N-n)=\lambda(t+1)^{-\alpha} d t,
$$

where $r / S$ has been replaced by $\lambda$ for notational convenience. Integrating both sides of this equation yields

$$
-\log (N-n)=\{\lambda /(1-\alpha)\}(t+1)^{1-\alpha}+C,
$$

where $C$ is the constant of integration. Multiplying both sides by -1 , exponentiating both sides, and rearranging produces

$$
n=N-\exp \left[-\{\lambda /(1-\alpha)\}(t+1)^{1-\alpha}-C\right],
$$

which can be reduced to

$$
n=N-k \exp \left[-\{\lambda /(1-\alpha)\}(t+1)^{1-\alpha}\right],
$$

where $k=\exp [-C]$. Note that $n=0$ when $t=0$ (i.e., no items have yet been recalled at the instant the recall period begins). Substituting these values into the above equation gives

$$
0=N-k \exp [-\lambda /(1-\alpha)],
$$

or

$$
N / k=\exp [-\lambda /(1-\alpha)] .
$$

Substituting $N / k$ for $\exp [-\lambda /(1-\alpha)]$ in Equation A1 yields

$$
n=N-k(N / k)^{(t+1)^{\beta}},
$$

where $\beta$ is equal to $1-\alpha$. This is Equation 6 in the text with $n=F(t)$. Note that when $t$ approaches $y$ and $\alpha$ is greater than 1 (i.e., $\beta$ less than 0 ), the above equation becomes $n=N-k$. That is, the number of items eventually recalled equals the number of items encoded $(N)$ minus the number forgotten during the course of recall $(k)$. Equation 5 is obtained by noting that the equation, $N / k=\exp [-\lambda /(1-\alpha)]$, can be rewritten as

$$
k=N \exp [\lambda /(1-\alpha)] \text {. }
$$

Substituting $N \exp [\lambda /(1-\alpha)]$ for $k$ in Equation A1 yields

$$
n=N\left(1-\exp \left[(\lambda / \beta)\left(1-(t+1)^{\beta}\right)\right],\right.
$$

which is Equation 5 with $n=F(t)$. Note that when $\alpha=0$ (such that $\beta=1$ ), which implies no forgetting, this equation reduces to the standard exponential growth function (i.e., Equation 1).

(Manuscript received July 21, 1993; revision accepted for publication October 27, 1993.) 